\title{
Measurement and Analysis of Degree of Position of Ball Track in Ball Screw Based on LabVIEW Xu Li and Jiang Yi
}

\author{
(NanChang Institute of Science and Technology,Nanchang,330108; Jiangxi Engineering Laboratory \\ of Digital Manufacturing for Automobile Parts)
}

Keywords: LabVIEW; Ball screw pair; Nut raceway; Measurement

\begin{abstract}
In order to solve the problem that the relative position of the looper hole and the spiral groove cannot be detected on site when the ball screw nut is processed at present, a method of converting the direct measurement into an indirect measurement method is proposed. Using software LabVIEW, a new type of ball is discussed. The method of measuring the position of the ball screw nut's inner raceway has established a ball screw nut's internal raceway position measuring system. Using this method, the position of the ball screw nut raceway was measured and compared with the theoretical value. The results show that the method has high measurement accuracy and reliability. The research results can lay a foundation for the precision measurement of the processing position of the return ball and the inner race of the nut.
\end{abstract}

\section{Introduction}

When processing internal recirculating ball screw nuts, it is common to machine the holes for mounting the retractor first, and then process the spiral grooves of the ball screw nut. When processing the spiral groove, the worker cannot determine the precise machining position of the spiral groove with respect to the returner hole, usually through trial grinding. The relative positional relationship between the returner and the spiral groove is determined by observing the direction of the spark. The processed returner hole observes the position of the spiral groove relative to the returner hole. This not only has a high technical requirement for the worker's operation, but also the precision of the position of the returning device hole and the spiral groove processed is difficult to guarantee, and the position of the returning device hole and the spiral groove directly affects the performance of the ball screw. The deviation of the position is larger. When the steel ball enters the returning device from the spiral groove or enters the spiral groove from the returning device, a sudden change occurs in the transition state of the steel ball. This mutation affects the precision of the ball screw movement; if the error is larger, , will cause steel ball jam phenomenon. Therefore, it is particularly important to accurately control and measure the position of the returner hole and the spiral groove. This paper proposes to use software LabVIEW software to adopt a new method of measuring the position of the inner raceway. This method only improves the inner race of the ball screw nut.

\section{Introduction to LabVIEW}

LabVIEW (Laboratory Virtual Instrument Engineering Workbench) is a graphical programming language development environment that is widely accepted by industry, academia, and research laboratories as a standard data acquisition and instrument control software. LabVIEW integrates all the functionality of hardware and data acquisition cards that meet the GPIB, VXI, RS-232, and RS-485 protocols. It also has built-in library functions that facilitate the application of software standards such as TCP/IP and ActiveX. This is a powerful and flexible software. Using it can easily establish your own virtual instrument, its graphical interface makes the programming and use process are vivid and interesting.

Graphical programming language, also known as "G" language. When programming in this language, the program code is basically not written and replaced by a flowchart or a block diagram. It uses as much as possible the terms, icons, and concepts familiar to technicians, scientists, and 
engineers. Therefore, LabVIEW is a tool for end users. It enhances your ability to build your own science and engineering system and provides an easy way to implement instrument programming and data acquisition systems. When using it for the principle research, design, testing and implementation of the instrument system, the work efficiency can be greatly improved.

Its main convenience is that, in the case of a hardware, the functions of different instruments can be realized by changing the software. It is very convenient and equivalent to software or hardware. It is a good software for accuracy measurement. This makes the measurement more convenient.

\section{Ball Screw Nut Pair}

The ball screw nut pair is a key function component on CNC machine tools and precision machinery, and it is characterized by high precision, reversibility, low frictional resistance and high efficiency. The ball screw pair is a screw groove in which the screw and the nut are screwed to place an appropriate number of balls as an intermediate transmission body. By means of the ball return passage, a spiral movement of the ball in a closed loop is repeated. The relative movement between the screw and the nut is changed from sliding friction to rolling friction by the action of the ball chain, which is also the most characteristic of the ball spiral movement. The ball screw is generally composed of four parts: screw, nut, ball, and ball return channel. Its appearance is shown in Figure 1. The ball screw nut pair is divided into an outer cycle and an inner cycle according to the cycle mode. The inner cycle structure is shown in Figure 2.

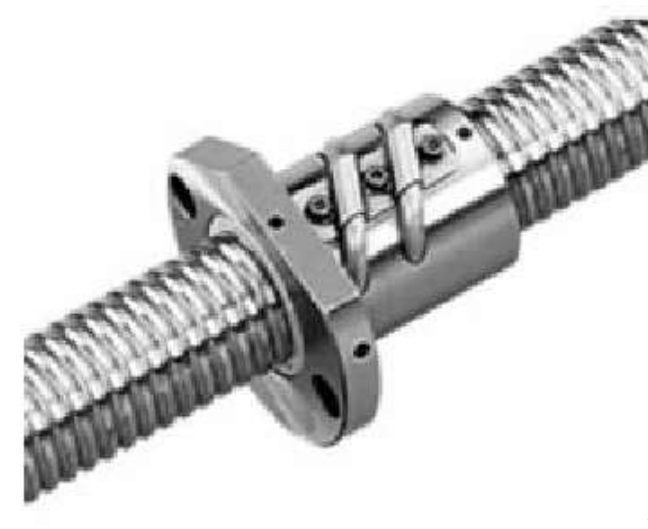

$\Delta$ Figure 1 Ball screw

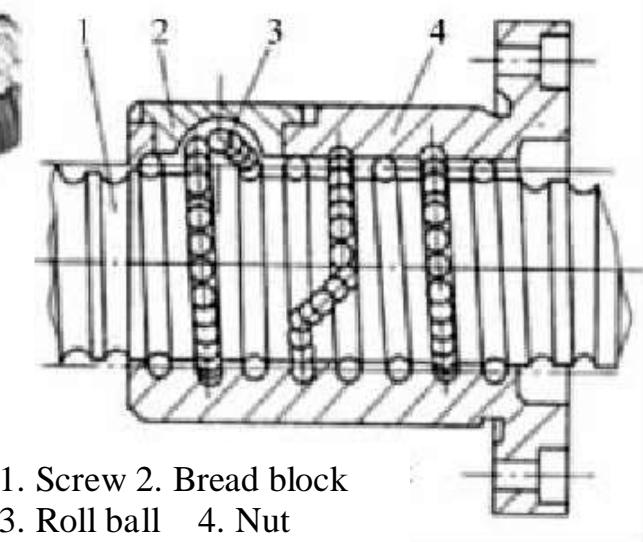

A Figure 2 Loop back

\section{Spiral Groove Position Measurement Principle}

In order to measure the relative position relationship between the returner hole and the spiral groove, determine whether the end of the returner hole and the spiral groove are tangent. The project team designed a measurement method. The measuring principle of this method is to directly measure the return device hole and The position error of the spiral groove is converted into the distance difference between the center of the spiral groove and the center point of the semicircular arc at the end of the return beads groove. If the difference between the ordinates on the helix line at the same angle can be obtained, the position error between the spiral groove and the return groove can also be obtained. When calculating the position error, first determine the center position of the spiral groove. When using it, hold the DUT 8 on the fixture 9 and use the two capacitive gate type digital dial gauges 6 and 5 as the measurement probe, one $x$ direction. The probe, a y-direction probe, the $\mathrm{x}$-direction probe and the digital dial indicator are at the ends of an equal-arm lever respectively, so that the displacement of the probe is consistent with the displacement of the digital display; the slide 2 can pass the screw 3 To slide up and down, the displacement in the y direction can be read out by pressing the dial indicator 5 on the carriage. In the measurement, first find a vertical part in the nut without a returner hole, and use the vertical probe of the instrument to pull up along the bottom of the inner surface of the ball screw nut to obtain the corresponding point coordinates. The normal 
section of the thread raceway is shown in Fig. 3. Within a certain error range, the projection curve of the spiral groove is fitted into a circular arc, and the corresponding circle center coordinates can be calculated.

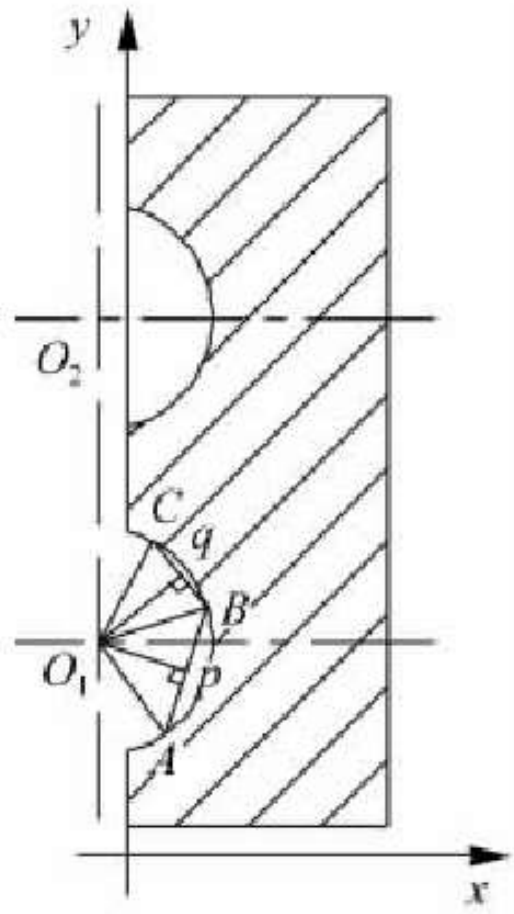

$\Delta$ Figure 3 Normal section of the thread raceway

\section{Ball Screw Nut Drive Should Pay Attention to the Use of Lubrication}

The ball screw nut transmission deputy should pay attention to lubrication, lubrication can improve the wear resistance of the transmission, to avoid the friction between the screw, nut, and the ball due to the application of a pre-tightening force to increase the part wear speed, reduce Produce backlash; Reduce the thermal deformation due to friction, improve machining accuracy; Lubrication can also improve transmission efficiency, so the lubrication of ball screw nut drive is very important.

Lubrication methods include grease lubrication and oil lubrication. Lubrication oil is generally used with turbine oil No. 90 180 (suitable for high-speed mechanical lubrication, mainly used for lubrication, cooling, and speed control), or spindle oil No. 140 Lubricating oil is generally injected into the space of the nut through the oil hole in the housing. Grease is mainly made of high-grade grease such as lithium base (having lubrication, anti-embroidering, and anti-pressure). The amount of grease contained in the bearing is generally lubrication space. 1/10. The enclosed volume of the ball screw nut transmission pair is generally $1 / 3$ of its internal space. Excessive grease contained will intensify friction and heat. After many years of operation of many machine tools, automatic screw-nut lubrication systems are often blocked and cannot be automatically lubricated. Adding advanced lithium-based grease can solve the problem and ensure the normal operation of bearings and screw nuts for several years.

\section{Conclusion}

For the current production line, there is no instrument that can quickly detect the position of the returner hole and the spiral groove. Using the precision measurement technology, the direct measurement is converted into indirect measurement to measure the relative position of the returner hole and the spiral groove. A new principle and method for measuring the position of internal raceway of ball screw nut is put forward, which can lay a foundation for the precision measurement of the position of the return ball and the internal raceway of the nut. Experiments show that this method has good feasibility and effectiveness. The research results can effectively solve the 
problem of measuring the position of return beads and spiral grooves in the production site of an enterprise, and have practical application value and economic value.

\section{Acknowledgement}

Project funding: Project at Nanchang institute of science and technology (No.SZZX-17-02)

\section{References}

[1] Petre M. Why Looking Isn't Always Seeing: Readership Skills and Graphical Programming.[J]. Communications of the Acm, 1995, 38(6):33-44.

[2] Zhang H, Zhou H, Guo X, et al. Study on Shaft Orbits Measurement and Identification Based on LabVIEW[J]. 2013:1087-1090.

[3] Wei Y, Xu W B, Zeng H J, et al. Rotor Axis Orbit Monitoring Based on Virtual Instrument[J]. Advanced Materials Research, 2011, 279:382-387.

[4] Zhang H X, Wang M Z, Li H, et al. Shaft orbit analysis based on LabVIEW for fault diagnosis of rotating machinery[C]// International Conference on Computer Science \& Education. IEEE, 2016.

[5] Zhang H X, Yan Y, Zhou H, et al. The Development of Virtual Experiment Platform for Signal Analysis and Processing Based on LabVIEW[J]. Applied Mechanics \& Materials, 2014, 513-517:3241-3244.

[6] Jr M S, Ghaghada K B, Badea C T, et al. A liposomal nanoscale contrast agent for preclinical CT in mice[J]. Ajr American Journal of Roentgenology, 2006, 186(2):300-7.

[7] Mccreary M Z. Synthesis of cadmium selenide quantum dots for fabrication of hybrid light emitting diodes $[\mathrm{J}]$. Dissertations \& Theses - Gradworks, 2014.

[8] Brown P, Beek T, Carr C, et al. Magnetoresistive magnetometer for space science applications[J]. Measurement Science \& Technology, 2012, 23(2):025902.

[9] Grzelczyk D, Stańczyk B, Awrejcewicz J. Prototype, control system architecture and controlling of the hexapod legs with nonlinear stick-slip vibrations[J]. Mechatronics, 2016, 37:63-78.

[10] Harasimowicz J, Welsch C P, Cosentino L, et al. Beam diagnostics for low energy beams[J]. Physical Review Special Topics - Accelerators and Beams, 2012, 15(12):42-47.

[11] Hurd J A, Sciver S W V. Measurements of the apparent thermal conductivity of multi-layer insulation between $20 \mathrm{~K}$ and $90 \mathrm{~K}[\mathrm{~J}]$. AIP Conference Proceedings, 2014, 1573(1):694-700.

[12] Gane L, Power S, Kushki A, et al. Thermal Imaging of the Periorbital Regions during the Presentation of an Auditory Startle Stimulus[J]. Plos One, 2011, 6(11):e27268. 